\title{
Reflexión crítica de los sistemas de gestión de calidad: ventajas y desventajas
}

\author{
Critical reflection of quality management systems: advantages and disadvantages
}

Recibido: 07-08-2019 • Aprobado: 11-08-2020 • Página inicial: 115 - Página final: 132

\author{
Yaritza Alejandra Murrieta Saavedra* \\ Eneida Ochoa Ávila** \\ Blanca Carballo Mendívil***
}

\begin{abstract}
Resumen: la calidad se ha aplicado desde tiempos remotos; sin embargo, la situación actual de las organizaciones ha hecho que las empresas implementen Sistemas de Gestión de Calidad (SGC) para incrementar la satisfacción del cliente y adquirir ventaja. El presente trabajo presenta una reflexión respecto a las ventajas y desventajas de los SGC, con el fin de ampliar la perspectiva del lector. Siguiendo la metodología de revisión de literaturas, se obtuvo que entre las ventajas se encuentran la profundización y mejora de procesos, la inclusión de aspectos ambientales y sociales, el refuerzo de la estructura organizacional y la confianza percibida por el cliente al adquirir productos de calidad; mientras que en las desventajas se detectan los altos costos, incremento en el número de empresas certificadas, mala toma de decisiones por certificarse rápidamente, el impedimento en la innovación y el desarrollo de nuevos productos, entre otras que se describen en el desarrollo del presente artículo.
\end{abstract}

Palabras clave: sistemas de gestión de calidad; SGC; gestión de la calidad; evolución.

\begin{abstract}
Quality has being practical for a long time. However, the current situation of organizations has forced companies apply Quality Management Systems (QMS) to increase costumer's satisfaction and get advantage over other companies. The following text was created from a compilation of information that describes the advantages and disadvantages of the QMS with the purpose of amplifying the reader's perspective. The result was that advantages have been found, for example, the deepening and improvement of processes, the inclusion of environmental and social aspects, the reinforcement of organizational structure and the confidence of the costumers when they buy quality products, but disadvantages have also been detected; high costs, increment of certified companies, wrong decisions taken because of the urgency for certification, impediments for innovation and development of new products, and some others described in this article.
\end{abstract}

Keywords: Quality management systems; QMS; quality management; evolution.

JEL: M19

* Maestra en Gestión Organizacional en el Instituto Tecnológico de Sonora (ITSON) de México. yaritzalems@gmail.com ORCID: http://orcid.org/0000-0002-9140-550X.

** Doctora en Planeación Estratégica para la Mejora del Desempeño y Doctora of Philosophy with a Major in Business Administration. Profesora investigadora de tiempo completo del Departamento de Psicología del Instituto Tecnológico de Sonora (ITSON) de México. Miembro del Sistema Nacional de Investigadores (SNI) nivel I. eneida.ochoa@itson.edu.mx ORCID: http://orcid.org/0000-0002-6510-8552.

*** Doctora en Planeación Estratégica para la Mejora del Desempeño. Profesora investigadora auxiliar del Departamento de Ingeniería Industrial del Instituto Tecnológico de Sonora (ITSON) de México. Miembro del Sistema Nacional de Investigadores (SIN) nivel C.

bcarballom@gmail.com

ORCID: http://orcid.org/0000-0003-0966-7146. 


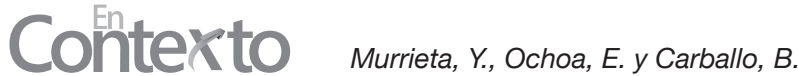

\section{Reflexão crítica dos sistemas de gestão qualidade: vantagens e desvantagens}

Resumo: a qualidade foi aplicada desde os tempos antigos; No entanto, a situação atual das organizações fez com que as empresas implementar Sistemas de Gestão da Qualidade (SGQ) para aumentar a satisfação do cliente e obter uma vantagem. Este artigo apresenta uma reflexão sobre as vantagens e desvantagens do SGQ, a fim de ampliar a perspectiva do leitor. Seguindo a metodologia de revisão de literatura, obteve-se que entre as vantagens estão o aprofundamento e melhoria dos processos, a inclusão dos aspectos ambientais e sociais, o reforço da estrutura organizacional e a confiança percebida pelo cliente na aquisição de produtos de qualidade; Enquanto as desvantagens são custos elevados, aumento do número de empresas certificadas, má tomada de decisão devido à rápida certificação, impedimento à inovação e ao desenvolvimento de novos produtos, entre outros que são descritos no desenvolvimento deste artigo.

Palavras-chave: sistemas de gestão de qualidade; SGQ; gestão de qualidade; evolução. 


\section{Introducción}

Los cambios económicos y de comercio internacional, ligados con las innovaciones tecnológicas y científicas, han dado dimensión al rol de la calidad en productos, procesos y servicios que hoy en día ofrecen las empresas, ya que los clientes han incrementado sus exigencias y esperan obtener el valor deseado por lo que están pagando. Es entonces que las empresas requieren de un adecuado sistema de gestión para una correcta toma de decisiones que les permita, no sólo cumplir los retos que sus clientes establecen (CarballoMendívil, Arellano-González y Ríos-Vázquez, 2018), sino aumentar su competitividad ante otras empresas, la cual es un indicador que se torna cada vez más complicado obtener ante la globalización, debido a que obliga a las empresas a tratar de sobresalir y posicionarse en el mercado de la aldea global (Priede, 2012).

Ahora bien, la calidad no es un concepto nuevo y ha sido ampliamente estudiado a través del tiempo por diversos personajes que son considerados los gurús de la calidad (Walter Shewhart, Joseph Jurán, Kaoru Ishikawa, Edward Deming, Armand Feigenbaum, Phil Crosby, Gen'ichi Taguchi, entre otros); en la época moderna los principios básicos establecidos por ellos se promueven por los Sistemas de Gestión de Calidad (SGC), que han sido situados como estrategias facilitan la implementación de prácticas que favorecen el logro de la competitividad en las empresas (Hernández, Stahnke y Núñez, 2004).

Es un hecho que la calidad se hace más importante para las organizaciones con el paso de los años, lo cual se puede constatar con el número de empresas que han buscado una certificación bajo los criterios de la normatividad emitida por la Organización Internacional para la Estandarización (ISO por sus siglas en inglés) o aplicado para un premio de excelencia, como el otorgado por la Fundación Europea para la Gestión de la Calidad (EFQM por sus siglas en inglés). Sin embargo, implementar un SGC no es un boleto seguro hacia la competitividad, por ello, antes de emprender este camino formal hacia la calidad a través de la implementación de un SGC, es importante que las organizaciones conozcan tanto los beneficios como las desventajas que estos conllevan.

Por lo anterior, el presente trabajo hace una revisión de la literatura de la evolución del concepto de calidad hasta los SGC; también plasma los beneficios y las debilidades de estos últimos, mediante una reflexión que se realiza con el objetivo de brindar al lector una perspectiva más amplia de los SGC, para el soporte en la toma de decisiones respecto a su implementación, dado que 


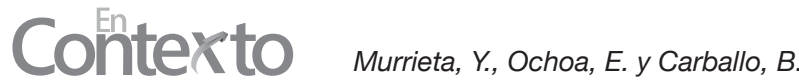

este es un elemento clave para el desarrollo de las organizaciones (Hernández, Barrios y Martínez, 2018).

\section{Desarrollo}

\section{Evolución del concepto de calidad}

La calidad se ha aplicado desde tiempos ancestrales, ya que los seres humanos siempre han requerido productos o servicios que cumplan con sus expectativas. Por ejemplo, en Egipto, con la construcción de las pirámides, ya se veían los sistemas de calidad, al sistematizar la construcción de las mismas para asegurar que estas cumplieran con lo estipulado (Pasi, 2013; Cruz-Medina, López-Díaz y Ruíz-Cárdenas, 2017).

Como se representa en la Figura 1, la calidad moderna aplicada en las organizaciones, en un inicio se centraba en disminuir los defectos de los productos; no obstante, con el tiempo se percató que requerían de una mayor atención de la administración de la calidad, para crear mejoras duraderas dentro de la organización y no solamente reducir los defectos de la misma (Evans y Lindsay, 2008).

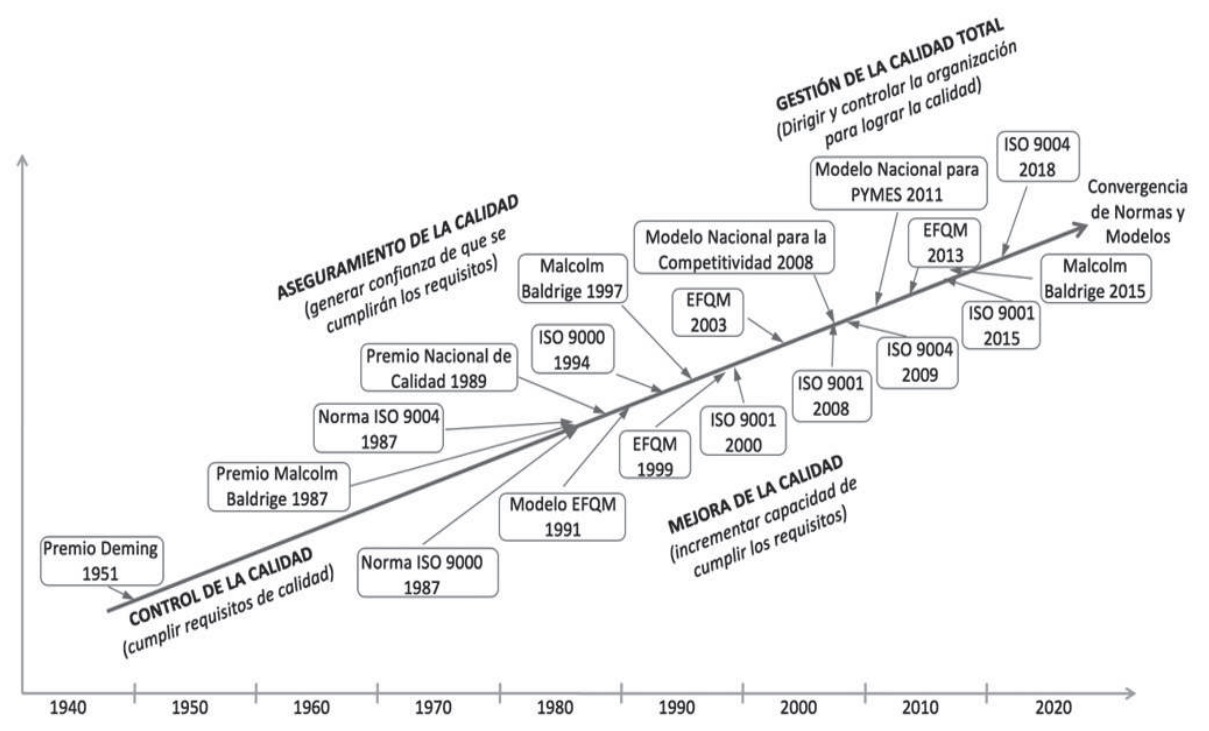

Figura 1. Evolución del concepto de la calidad a través de cambios en normas y modelos. Elaboración propia. 
Entre 1900 y 1940 la calidad consistía en excluir aquellos productos terminados que tenían defectos y enviar al cliente el resto del producto de calidad (Weckenmann, Akkasoglu \& Werner, 2015). Ciertamente, este proceso implicaba desperdicios que eran traducidos en pérdidas de dinero. A partir de entonces, comenzó la evolución de la calidad como un método que gestionaba a las organizaciones, surgió primeramente con el control de la calidad y tuvo sus orígenes en Japón, en el año 1950, y germó sus principios en Toyota (Dahlgaard \& Dahlgaard-Park, 2006).

Específicamente durante las décadas de 1910 y 1920, la gestión de la calidad, que en ese momento era exclusivamente industrial, estaba limitada a la inspección de productos terminados y la eliminación de los artículos defectuosos; hasta que, en 1924, el estadounidense Shewhart (1931) sentó las bases del Control Estadístico de Procesos, el cual remarcó la importancia de reducir la variación en un proceso de manufactura para evitar las no conformidades.

A principios de la década de 1950, Ishikawa (1997) fue un gran promotor del control de calidad al estilo japonés, contribuyendo al desarrollo del país después de la Segunda Guerra Mundial. Este teórico fue quien destacó que el estilo de calidad japoneses y occidentales son diferentes, no sólo por sus discrepancias culturales, religiosas y de educación, sino porque ellos no fueron influenciados por los principios de la Administración Científica de Taylor. El enfoque de control de calidad que promovió, se caracterizó por la participación de todos, desde la Alta Dirección hasta los operadores; y su principal aporte técnico fue el desarrollo de los métodos estadísticos prácticos y accesibles para ese tiempo: las siete herramientas básicas de la administración de la calidad, dentro de las cuales se incluía el aporte de Shewhart (1931), definidas como una filosofía administrativa que se encuentran detrás de la calidad a través de 11 principios que señalan la importancia de la educación en la misma, el conocimiento de los clientes, la supresión de los defectos desde la raíz lo que hace posible a su vez la eliminación de la inspección, la participación de toda la organización en el proceso, enfatizan la resolución de problemas mediante el análisis y hacen alusión de la variabilidad como punto de validez de los datos.

Simultáneamente, se hacía relevante la aportación de Jurán (1951) y su publicación del libro Quality Control Handbook, reconociéndose como uno de los principales manuales para la calidad útiles en la práctica y sugiere tres procesos: la planeación, el control y la mejora de la calidad. 


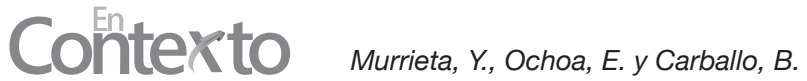

En esta misma década, el estadounidense Deming (1986) llegó a Japón para apoyar en la educación de temas de control estadístico de procesos y otros conceptos de calidad propuestos por Shewhart (1931), como el ciclo Plan-DoCheck-Act (PHVA por sus siglas en español), y propuso crear en este país un premio para las empresas que demostraran un comportamiento ejemplar en la mejora de calidad: el premio Deming, cuyo proceso de auditoría propuso Ishikawa. Con esta experiencia estableció 14 principios que posteriormente aplicó en Estados Unidos para su conversión industrial hacia el aseguramiento de la calidad; estos principios hacen hincapié en la mejora continua, la capacitación, el liderazgo en los directivos, el involucramiento de toda la organización, la integración de la calidad en todo el proceso productivo y a la transformación conjunta.

En este tiempo, el estadista japonés Taguchi desarrolló métodos para mejorar la calidad y la fiabilidad, estableciendo innovaciones en el tema del diseño de experimentos, e indicando que es más barato rediseñar los procesos de fabricación y productos que realizar acciones de mejora de la calidad tras una auditoría (Taguchi, Chowdhury \& Wu, 2005).

Para 1960, aparecen otros personajes como Feigenbaum (1991), quien creó el concepto del Control Total de la Calidad, con lo que definió el concepto de calidad con un enfoque sistémico, es decir, no sólo como responsabilidad del departamento de producción, sino de toda la organización, orientando así el control de la calidad total hacia la excelencia, y no hacia los defectos como se venía realizando. Este nuevo enfoque, que luego se conoció como Administración de Calidad Total, influyó profundamente en la competitividad de los mercados nacionales e internacionales, especialmente en Estados Unidos y Japón.

Dada esta situación, en la década de 1980, en Estados Unidos se vive una crisis relacionada con la calidad, debido a la pérdida de mercados frente a los productos japoneses, dada la superioridad de estos últimos. En este tiempo aparece Crosby (1979) para dar respuesta a la crisis con su máxima: doing it right the first time (DIRFT), que significa "hacerlo correctamente la primera vez", y otros 14 principios que se consideran como una receta para el impulso de la calidad, bajo la creencia de que la implementación de este ciclo continuo de gestión de calidad tendría más ahorros en los costos que en los gastos en que incurriría, estableciendo que la calidad es gratis y focalizando en el compromiso, medición, mejora continua, capacitación y reconocimiento. 
Posteriormente, a finales de los años ochenta surgió la etapa del aseguramiento de la calidad con el nacimiento de premios y modelos que garantizaban la calidad del producto desde los procesos. En las empresas estadounidenses, se encontraba el Malcom Baldrige National Quality Award, mientras que en Europa apareció el EFQM; lo que generó competitividad entre las empresas (Mangelsdorf, 1999) y creó el marco de referencia para la Gestión de la Calidad Total (TQM por sus siglas en inglés) (Yusof \& Aspinwall, 2000).

Esta tendencia organizacional de premios y modelos de la calidad tuvo como resultado una expansión a nivel mundial. En 1989, en México se establece el Premio Nacional de la Calidad (PNC) que dio paso a la creación del Instituto para el Fomento de la Calidad Total (IFC) y brindó, después, un modelo de referencia a nivel nacional para la gestión de la calidad de las empresas mexicanas (Premio Nacional de la Calidad, 2019).

La implementación de la norma ISO, emergió como la tercera fase en la transición del control de la calidad a la gestión de la calidad (Mangelsdorf, 1999), creando la responsabilidad entre los gerentes de la calidad de documentar, regular las actividades y de estandarizar (Weckenmann et al., 2015).

Lo previamente mencionado consolida la cuarta etapa de transformación de la calidad: la TQM, considerada una filosofía holística de gestión de la calidad, cuyo propósito es "cambiar la cultura corporativa, de una pasiva y defensiva cultura a una proactiva y abierta, donde los principios básicos de la TQM incrementan la satisfacción del cliente e implementen una continua participación de todos los que integran la organización" (Dahlgaard \& Mi Dahlgaard Park, 2006, p.273).

Ahora bien, dado el propósito del presente artículo, se hace necesaria la expansión del concepto de SGC como elemento que permite la TQM, lo cual puede ser definido como una estrategia que tiene como fin capacitar a los altos mandos sobre los sistemas que deben implementar en su empresa, con el propósito de aumentar la satisfacción de sus clientes y adquirir ventaja competitiva (Stahl \& Grigsby, 1997). Este sistema trae consigo una filosofía que busca no sólo la supervivencia de la organización, sino su crecimiento, y se centra en la mejora de la calidad de sus productos, servicios y la organización en total (James, 1997).

En adición a esto, la Sociedad Americana de Calidad define un SGC como un “(...) sistema formal que documenta la estructura, procesos, roles, 


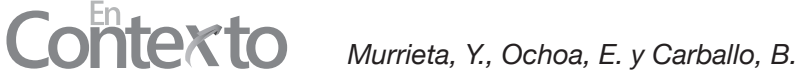

responsabilidades y procedimientos requeridos para lograr una gestión de calidad efectiva" (American Society for Quality, 2018). En relación a las definiciones anteriores, se podría deducir que un sistema de gestión de calidad es un conjunto de acciones, que busca la satisfacción de los clientes y la calidad de los productos o servicios mediante una documentación formal, procesos, roles y responsabilidades.

La popularidad alcanzada de los SGC ha permitido su aplicación en áreas y sectores no industriales; en el área de la salud Betlloch-Mas, Ramón-Sapena, Abellán-García y Pascual-Ramírez (2019) sugieren que la certificación proporciona mayor comodidad y seguridad para los clientes; en el área educativa, la planeación y el aprendizaje organizacional permiten ofrecer una enseñanza de calidad. A pesar de estos casos, el camino por recorrer en el área es largo debido a sus implicaciones institucionales (Díez, Villa, López \& Iraurgi, 2020).

\section{Ventajas de implementar un Sistema de Gestión de Calidad}

Los beneficios que tiene implementar un SGC se reportan en la investigación realizada por Pasi (2013), quien manifiesta que este tipo de sistemas toma en cuenta el ambiente y la sociedad, consecuentemente, resulta innovador y adaptables a las tecnologías que se encuentran en tendencia. A su vez, expresa que las futuras generaciones continuarán desarrollando SGC, lo cual enriquecerá la calidad de los productos o servicios que las empresas ofrecen.

De igual forma, Hernández (2009) enuncia que las organizaciones que efectúan dichos sistemas, desarrollan la capacidad de energía sinérgica al momento de involucrar en la organización al personal, a su entorno, a la sociedad, a los proveedores y sus clientes, abriendo camino a transformarse en organizaciones inteligentes. Además, tienden a profundizar en sus procesos y en el conocimiento de los mismos, delimitando las responsabilidades de los que conforman la organización y su función en el desarrollo de los mismos. A esto se suma, que sus empleados adquieren conciencia de la importancia de la calidad; la empresa adopta la capacidad de conocer las expectativas de los clientes, deseos de los consumidores y, por último, la reducción de los costos y el incremento de las ventas (Álvarez, Fraiz y Del Río, 2013).

Para que una organización sobresalga de otras, existen diversas estrategias como el ofrecer precios bajos, lo cual a largo plazo resulta desfavorable para la empresa, o incrementando la calidad del servicio que ofrecen, mediante la implementación de estrategias de TQM, con el fin de ofrecer un mejor producto 
o servicio con base en la mejora de sus procesos e incrementar la satisfacción de los clientes y de los involucrados. Por lo tanto, gestionar a través de la calidad resulta ser una práctica sobresaliente para adquirir ventaja competitiva (Kit, Shahar \& Akmar, 2018).

Un estudio empírico, realizado por Benzaquen-De Las Casas y PérezCepeda (2016), aporta a la literatura beneficios que obtienen las empresas que implementan un sistema de gestión de calidad, en este caso ISO 9001, en comparación con las empresas que no están certificadas en la norma, encontrando diferencias significativas en aquellas empresas certificadas, sobresaliendo en los elementos de las estrategias de la TQM (Liderazgo, Planeamiento de la Calidad, Auditoría y Evaluación de la Calidad, Diseño del Producto, Gestión de la Calidad del Proveedor, Control y Mejoramiento del Proceso, Educación y Entrenamiento, Círculos de la Calidad y Enfoque hacia la Satisfacción del Cliente). Aunado a esto se hace inmerso que las empresas que están bajo la normativa de certificación de los SGC tienen un nivel de madurez mayor, indicando evolución en los procesos y mejora continua (Rohvein et al., 2019).

Asimismo, Lizarzaburu (2015) aporta variedad de ventajas al implementar el sistema de gestión ISO 9001, entre ellas sobresalen la mejora continua, la transparencia en los procesos, el aseguramiento de los objetivos establecidos por la organización, la satisfacción y tranquilidad de los clientes, el aumento de la eficiencia y la productividad y, por último, beneficios financieros tangibles para la empresa.

Por último, Arraut (2010) considera que los SGC contribuyen a la innovación de las organizaciones, siendo una herramienta fundamental para el crecimiento económico de las naciones haciéndolas más productivas, ofreciendo productos y servicios en menor tiempo, con menores costos y mayor calidad, generando una alta competitividad a las empresas.

\section{Desventajas de implementar un Sistema de Gestión de Calidad}

No obstante, a los beneficios de la implementación de los SGC, se encuentran aspectos relevantes en cuanto a las limitaciones, debilidades o desventajas de los sistemas de calidad. Estos factores se pueden resumir en costos, desventajas de los sistemas y las dificultades que existen dentro de las organizaciones que no permiten resultados favorables al implementarlos. 


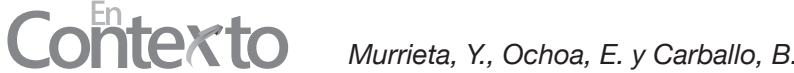

Año tras año, como se mencionó anteriormente, las empresas optan por implementar estrategias competitivas, en especial, el incremento de la calidad de los servicios o productos mediante SGC, lo cual ha impactado a los altos números de empresas certificadas en las normas ISO 9001. Para Priede (2012), en el año 2010, más de un millón de compañías y organizaciones estaban certificadas de acuerdo a los estándares de dicha norma. Estas cifras ponen en controversia la verdadera percepción de los SGC como estrategias que aportan ventaja competitiva.

Igualmente, esta situación de incremento de empresas certificadas, ha retado a las organizaciones que no están certificadas con la norma, y que en algunas ocasiones sus servicios y productos resultan ser de calidad; sin embargo, la situación económica impide la certificación por los altos costos que implica certificarse en la norma ISO 9001. Específicamente en México, donde la situación económica no es tan favorable como en otras naciones, el certificarse en la norma cuesta alrededor de 5000 dólares (Zamora, 2015), esto sujeto a cuestiones tales como el tamaño de la empresa y su giro (Sánchez, 2018).

Aunado a los gastos que implica la certificación, cuando la empresa no cuenta con expertos en la norma, requieren la contratación de consultores externos que aporten el servicio a la empresa y ayuden a la certificación, generando un costo extra, además de los gastos de capacitación del personal. Por otra parte, debido al incremento de empresas que buscan la certificación de la norma, aumentan también las entidades que ofrecen el servicio de consultoría, trayendo consigo que, las empresas por reducir gastos de inversión, opten por contratar consultores, los cuales no siempre resultan ser los más capacitados para este tipo de trabajo, o bien, debido a las exigencias de la empresa y reducción de tiempos, no realicen este tipo de proceso de manera adecuada y de algún modo terminen certificándose en la norma ISO (Brzozowski, Rogala \& Skowron, 2014).

De la misma manera, algunas organizaciones tienden a certificarse por el hecho de cumplir un requisito, y no con el fin de implementar la calidad del servicio que aportan, olvidando la concientización del personal sobre la misma. Cuando los directivos no reconocen las ventajas de los SGC, no pueden aportar ni trasmitir a sus subordinados esta cultura de calidad, haciendo que los resultados de los SGC resulten desfavorables para la empresa (Heras-Saizarbitoria, Casadesús \& Marimón, 2011; Martínez y Martínez, 2008). Rogala (2011) señala que las dificultades de encontrar beneficios en los SGC, se debe a problemas de comunicación interna, cumplimento de objetivos y falta de conciencia entre los empleados. 
Entre las desventajas de los SGC, se encuentra su aplicación limitada para los tipos de organizaciones. Mientras que Lara (2002), identifica que estos sistemas son aplicables entre las organizaciones de giro de servicios, Heras-Saizarbitoria et al. (2011) indica que aplicar un SGC a las organizaciones de servicios tiene menores beneficios que los encontrados en las empresas que ofrecen productos.

Del mismo modo, Vicher-García (2012) señala que existen diferencias en la aplicación de la norma ISO, en cuanto a si la empresa es de carácter público o privado, manifestando que la norma se funda por ingenieros y su principal enfoque es hacia organizaciones privadas. Sin embargo, debido a la popularidad de los SGC, se han expandido hacia organizaciones públicas, en ocasiones, trayendo resultados desfavorables a los objetivos establecidos, ya que, para las instituciones públicas burocráticas, que ofrecen servicios al ciudadano, implica tiempo los requisitos de documentación, ocasionando que se dejen de lado las actividades diarias propias de la organización, y teniendo resultados negativos en el servicio al ciudadano.

Por otra parte, se encuentran controversias en cuanto a la efectividad de la empresa y su relación con los SGC. Un estudio realizado por Boiral \& Amara (2009) destaca que para algunas empresas se atañen a un mayor desempeño empresarial, pero altos problemas internos, y otro grupo de empresas presentan alto desempeño y bajos problemas internos.

De la misma forma, mientras que Arraut (2010) asocia los SGC con la innovación, Malaver, Cardona y Rivera (2010) formulan que este tipo de gestión limita a las empresas en la innovación y desarrollo de nuevos productos, haciendo referencia específicamente a ISO. Esto en concordancia con lo señalado por Porter (1996), quien expresa que lejos de ser estrategias para crear ventaja competitiva, resultan ser ineficacias operacionales y no aportan diferenciación a la organización; por ende, el posicionamiento no es resultado de su aplicación. Debido a la rápida expansión de las mejores prácticas, las empresas difícilmente obtienen posicionamiento prolongado en el mercado, en consecuencia, los SGC no resultan ser sinónimo de estrategia.

En este mismo orden de ideas, DiMaggio \& Powell (1983) aluden que las organizaciones, conforme avanza su tiempo en el mercado, tienden a ser homogéneas en procesos y comportamiento, a lo que llaman isomorfismo. Así pues, el isomorfismo mimético, específicamente, se da cuando existe ambigüedad en los procesos y las organizaciones optan por imitar a la competencia con mayor tiempo en el mercado, esto lleva a la rápida proliferación de técnicas para igualar el éxito de los sistemas japoneses y europeos principalmente. 


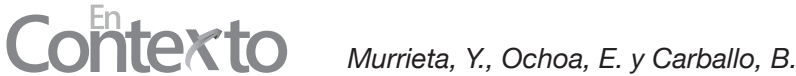

Por último, los SGC, como ISO, establecen normas que deben seguir las organizaciones para obtener certificación en sus procesos. Esto, en relación a lo expuesto por Evan (1967), las organizaciones de referencia normativa limitan a las empresas en la toma de decisiones, lo que podría resultar impedimento cuando se presentan contingencias dentro de la organización.

En síntesis, las ventajas y desventajas de los SGC, se presentan en la Tabla 1, la cual sintetiza lo expuesto en el desarrollo del presente artículo, englobando los aspectos analizados y permitiendo una mejor comprensión para el lector.

Tabla 1.

Ventajas y desventajas de implementar un $S G C$

\begin{tabular}{ll}
\hline \multicolumn{1}{c}{ Ventajas } & \multicolumn{1}{c}{ Desventajas } \\
\hline & - Alto costo. \\
& - Incremento de empresas \\
& certificadas, lo que ya no aporta \\
- Obliga a las organizaciones a tomar en & ventaja competitiva. \\
cuenta aspectos ambientales y sociales. & - La necesidad de certificarse puede \\
- Profundización en procesos. & llevar a tomar malas decisiones. \\
- Procesos documentados. & En ocasiones se cumple solo \\
- Refuerza la estructura organizacional. & por requisito y no se le da la \\
- Energía sinérgica. & importancia necesaria. \\
- Confianza y seguridad al cliente. & No es una estrategia como tal. \\
- Mejora en procesos. & - Limita la toma de decisiones. \\
- Eficiencia. & Crea organizaciones homogéneas. \\
- Productividad. & Inhibe la innovación y desarrollo \\
- Madurez en la organización. & de nuevos productos. \\
& - Su relevancia no es comprobable \\
& para todos los sectores. \\
\hline
\end{tabular}

Elaboración propia.

\section{Conclusiones}

Una vez analizadas las desventajas y desventajas de los SGC, se concluye que la eficacia de aplicar un SGC en una organización depende de diversas variables que deben ser tomadas en cuenta al momento de decidir su implementación; entre ellas se encuentran la fortaleza de la estructura organizacional y la comunicación existente dentro de la misma. 
La popularidad de gestionar las organizaciones con base en la calidad, limita a las organizaciones en la investigación empírica en diversos sectores, considerando la diversidad de procesos existentes entre los mismos. Dicho esto se recomienda a los interesados del éxito de las empresas realizar un análisis exhaustivo de las características de la misma, en cuanto a situación actual de recursos financieros y humanos, los objetivos y metas de la organización, quiénes son sus clientes y a quien dirigen su producto o servicio, antes de decidir la implementación de un SGC, para posteriormente reflexionar si es necesario aplicar el sistema o si existe otro método para gestionar la organización en torno a otros elementos, como el recurso humano o los clientes.

Los SGC no pueden ser considerados una estrategia si se trabajan de manera única, para que cumplan la función de estrategia, y obtener ventaja competitiva, debe ser la calidad el elemento diferenciador de la organización, o bien, la visión apunta a distinguirse por ofrecer un producto o servicio de calidad. Además, deberá ser acompañado de un análisis del contexto interno y externo y de otras herramientas con enfoque de TQM.

En la actualidad, la tendencia en los sistemas de gestión implica la integración de los sistemas en uno solo, y no como tradicionalmente se venía manejando. Esto lo vemos desde el año 2015, cuando ISO comenzó a estandarizar la estructura documental de sus normas certificables (9001, 14001 y 45001), de tal manera que pudieran interconectarse entre ellas para funcionar como un todo, tal como se había pregonado a través de los principios de calidad que incluían el enfoque de sistemas como la clave para la obtención de resultados organizacionales esperados y la competitividad.

El presente artículo contribuye también a la investigación empírica, abriendo campo a posibles estudios respecto a los sistemas de gestión empresarial, ya que se observan algunas controversias entre autores respecto a las ventajas de los SGC. Primero, se encuentra una disyuntiva en el impacto de la aplicación de los sistemas de gestión de calidad y su relación con el tipo de empresa; por otra parte, investigar si los SGC, en especial la norma ISO, están positivamente relacionados con la innovación de nuevos productos y con la comunicación interna.

Por último, se recomienda ampliar la búsqueda de información en artículos empíricos, para encontrar mayor información que apoye las ventajas y desventajas expuestas en el presente trabajo, o bien, las rechace. De este modo, se permitirá expandir las perspectivas del lector y así, facilitar la toma de decisiones asertivas encaminadas al éxito de las organizaciones. 


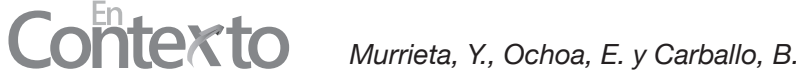

\section{Referencias}

Álvarez, J., Fraiz, J. y Del Río, M. (2013). Implantación de un sistema de gestión de la calidad: beneficios percibidos. Revista Venezolana de Gerencia (RVG), 18(63), 379-407. Recuperado de http://www.redalyc. org/articulo.oa? $\mathrm{id}=29028476002$

American Society for Quality. (2018). Quality glossary. Recuperado de https:// asq.org/quality-resources/quality-glossary

Arraut, L. (2010). La gestión de calidad como innovación organizacional para la productividad en la empresa. Revista Escuela de Administración de Negocios, 69, 22-41. doi: https://doi.org/10.21158/01208160. n69.2010.515

Benzaquen-De Las Casas, J. y Pérez-Cepeda, M. (2016). El ISO 9001 y TQM en las empresas de Ecuador. Revista Globalización, Competitividad, 10(3), 153-176. doi: https://doi.org/10.3232/GCG.2016.V10.N3.06

Betlloch-Mas, I., Ramón-Sapena, R., Abellán-García, C. \& PascualRamírez, J. (2019). Implementation and Operation of an Integrated Quality Management System in Accordance with ISO 9001:2015 in a Dermatology Department. Actas Dermo-Sifiliográficas (English Edition). doi: 10.1016/j.adengl.2019.01.003

Boiral, O. \& Amara, N. (2009). Paradoxes of ISO 9000 Performance: A Configurational Approach. The Quality Management Journal, 16(3), 3660. doi: https://doi.org/10.1080/10686967.2009.11918240

Brzozowski, T., Rogala, P. \& Skowron, P. (2014). Quality management system ISO 9001-weaknesses, practical irregularities and threats. Recuperado de https://www.tvp.zcu.cz/cd/2014/PDF_sbornik/brzozowski\%20rogala\%20skowron.pdf

Carballo-Mendívil, B., Arellano-González, A. y Ríos-Vázquez, N. (2018). La gestión de procesos esbeltos como principio de mejora. Un caso aplicado a una comercializadora. 3C Empresa: Investigación y pensamiento crítico, 7(3), 60-81. doi: http://dx.doi.org/10.177993/3cemp.2018.070335.60-81/

Crosby, P. (1979). Quality is free: the art of making quality certain. United States: McGraw-Hill. 
Cruz-Medina, F., López-Díaz, A. y Ruiz-Cárdenas, C. (2017). Sistema de gestión ISO 9001-2015: técnicas y herramientas de ingeniería de calidad para su implementación. Ingeniería, Investigación y Desarrollo, 17(1), 59-69. doi: https://doi.org/10.19053/1900771X.v17.n1.2017.5306

Dahlgaard, J. \& Mi Dahlgaard-Park, S. (2006). Lean production, six sigma quality, TQM and company culture. The TQM Magazine, 18(3), 263281. doi:10.1108/09544780610659998

Deming, W. (1986). Out of the Crisis. United States: MIT Press.

Díez, F., Villa, A. Lopez, A. \& Iraurgi, I. (2020). Impact of quality management systems in the performance of educationalcenters: educational policies and management processes. Heliyon, 6(4), 1-7. doi: https://doi. org/10.1016/j.heliyon.2020.e03824

DiMaggio, P. \& Powell, W. (1983). The Iron Cage Revisited: Institutional Isomorphism and Collective Rationality in Organizational Fields. American Sociological Review, 48(2), 147-160. doi: 10.2307/2095101

Evan, W. (1967). La órbita de la organización: Hacia una teoría de las relaciones interorganizacionales. En J. Thompson (Comp.), Teoría de la organización. Buenos Aires, Argentina: Bibliográfica Omeba.

Evans, J. y Lindsay, W. (2008). Administración y control de calidad. México: Cengage Learning.

Feigenbaum, A. (1991). Total Quality Control. New York, United States: McGraw-Hill.

Heras-Saizarbitoria, I., Casadesús, M. \& Marimón, F. (2011). The impact of ISO 9001 standard and the EFQM model: The view of the assessors. Total Quality Management \& Business Excellence, 22(2), 197-218. doi: $10.1080 / 14783363.2010 .532330$

Hernández, A. (2009). Gestión de la calidad y gestión del conocimiento. Boletín Científico Técnico INIMET, 2, 28-33. Recuperado de http://www.redalyc. org/pdf/2230/223018305004.pdf

Hernández, H., Barrios, I. y Martínez, D. (2018). Gestión de la calidad: elemento clave para el desarrollo de las organizaciones. Criterio Libre, 16(28), 179-195. 


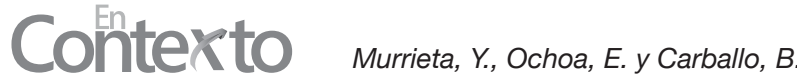

Hernández, J., Stahnke, W. y Nuñez, S. (2004). Sistemas de gestión de la calidad en el sector agroalimentario. AGROALIMENTARIA, 18, 87-93. Recuperado de http://erevistas.saber.ula.ve/index.php/agroalimentaria/ article/view/1317/1264

Ishikawa, K. (1997). ¿Qué es el control total de calidad?: la modalidad japonesa. Barcelona, España: Editorial Norma.

James, P. (1997). La gestión de la calidad total: Un texto introductorio. Madrid, España: Prentice Hall.

Jurán, J. (1951). Quality Control Handbook. New York, United States: McGraw-Hill.

Kit, S., Shahar, M. \& Akmar, N. (2018). The impact of total quality management (TQM) on competitive advantage: a conceptual mixed method study in the Malaysia Luxury Hotel Industries. Academy of Strategic Management Journal, 17(2), 1-9. Recuperado de https://www.abacademies.org/ articles/the-impact-of-total-quality-management-tqm-on-competitiveadvantage-a-conceptual-mixed-method-study-in-the-malaysia-luxuryhotel-i-7115.html

Lara, J. (2002). La gestión de la calidad en los servicios. Conciencia Tecnológica, 19, 1-5. Recuperado de http://www.redalyc.org/pdf/944/94401905.pdf

Lizarzaburu, E. (2015). La gestión de la calidad en Perú: un estudio de la norma ISO 9001, sus beneficios y los principales cambios en la versión 2015. Universidad \& Empresa, 18(30), 33-54. doi: dx.doi.org/10.12804/rev. univ.empresa.30.2016.02

Malaver, M., Cardona, D. y Rivera, H. (2010). La implementación de las tecnologías de gestión de calidad y su relación con la innovación. Pensamiento y gestión, 29, 104-123. Recuperado de http://rcientificas. uninorte.edu.co/index.php/pensamiento/article/view/1972/4979

Mangelsdorf, D. (1999). Evolution from quality management to an integrative management system based on TQM and its impact on the profession of quality managers in industry. The TQM Magazine, 11(6), 419-425. doi:10.1108/09544789910287737 
Martínez, M. y Martínez, Á. (2008). Sistemas de gestión de calidad y resultados empresariales: una justificación desde las teorías institucionales y de recursos y capacidades. Cuadernos de Economía y Dirección de la Empresa, 11(34), 7-30. doi: https://doi.org/10.1016/S11385758(08)70051-3

Pasi, Y. (2013). Development of quality management systems: how have disruptive technological innovations in quality management affected organizations? Quality innovation prosperity, XVII(1), 104-119. doi: 10.12776/QIP.V17I1.154

Porter, M. (1996). What is strategy?. Harvard Business Review, 74(6), 61-78.

Premio Nacional de la Calidad (PNC). (2019). Nuestra historia. Recuperado de https://www.pnc.org.mx/nuestra-historia-2/

Priede, J. (2012). Implementation of Quality Management System ISO 9001 in the World and its Strategic Necessity. Procedia-Social and Behavioral Sciences, 58, 1466-1475. doi: 10.1016/j.sbspro.2012.09.1133

Rogala, P. (2011). Problems related to maintaining and improving ISO 9001 Quality Management System. Recuperado de https://dspace.tul.cz/bitstream/handle/15240/21296/ACC_2011_3_14.pdf?sequence=1\&isAllowed $=\mathrm{y}$

Rohveinl, C., Jaureguiberry, M., Urrutia, S., Roark, G., Chiodi, F. y Paravie, D. (2019). Modelo de madurez como base para el diagnóstico de la gestión de procesos pyme. Revista Ingeniería Industrial, 18(1), 5-26. Recuperado de http://revistas.ubiobio.cl/index.php/RI/article/view/4069/3804

Sánchez, T. (2018). Costo/ beneficio en la implementación un sistema de gestión de calidad para las pymes (Tesis de pregrado). Universidad Militar Nueva Granada, Bogotá, Colombia.

Shewhart, W. (1931). Economic Control of Quality of Manufactured Product. New York, USA: Van Nostrand Company, Incorporated.

Stahl, M. \& Grigsby, D. (1997). Strategic Management. Total Quality \& Global Competition. Oxford: Wiley. 


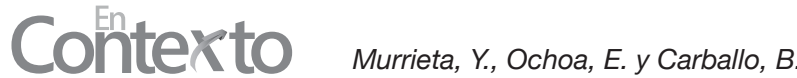

Taguchi, G., Chowdhury, S. \& Wu, Y. (2005). Taguchi's Quality Engineering Handbook. United States: Wiley.

Vicher-García,M.(2012). Utilidad o futilidad: calidad e ISOs en la administración pública.Convergencia, 19(60),205-228.Recuperadodehttp://www.scielo. org.mx/scielo.php?script=sci_arttext\&pid=S1405-14352012000300007

Weckenmann, A., Akkasoglu, G. \& Werner, T. (2015). Quality management - history and trends. The TQM Journal, 27(3), 281-293. doi:10.1108/ tqm-11-2013-0125

Yusof, S. \& Aspinwall, E. (2000). Total quality management implementation frameworks: Comparison and review. Total Quality Management, 11(3), 281-294. doi:10.1080/0954412006801

Zamora, A. (2015). Evaluación de tres directrices para la implementación de un sistema de gestión de la calidad. Revista latinoamericana de patología clínica, 62(1), 11-15. Recuperado de https://www.medigraphic.com/ pdfs/patol/pt-2015/pt151c.pdf

\section{Para citar este artículo:}

Murrieta, Y., Ochoa, E. y Carballo, B. (2020). Reflexión crítica de los sistemas de gestión de calidad: ventajas y desventajas. EnContexto, 8(12), 115-132. 